University of Wollongong

Research Online

University of Wollongong in Dubai - Papers

University of Wollongong in Dubai

2006

\title{
The slope line code for digital communication systems
}

Abdullatif Glass

Technical Studies Institute, UAE

Nidhal Abdulaziz

University of Wollongong in Dubai, nidhal@uow.edu.au

Eesa Bastaki

Dubai Silicon Oasis

Follow this and additional works at: https://ro.uow.edu.au/dubaipapers

\section{Recommended Citation}

Glass, Abdullatif; Abdulaziz, Nidhal; and Bastaki, Eesa: The slope line code for digital communication systems 2006.

https://ro.uow.edu.au/dubaipapers/236

Research Online is the open access institutional repository for the University of Wollongong. For further information contact the UOW Library: research-pubs@uow.edu.au 


\section{The Slope Line Code for Digital Communication Systems}

\author{
Abdullatif Glass \\ Department of Planning and Training \\ Technical Studies Institute \\ P O Box 26833, Abu-Dhabi, UAE \\ Tel: +9712 5045785 , Fax: +97125854282 \\ e-mail: aglass@ieee.org
}

\author{
Nidhal Abdulaziz \\ College of Information Technology \\ University of Wollongong in Dubai \\ P O Box 20183, Dubai, UAE \\ Tel: +971 4 3672490, Fax: +971 43672754 \\ e-mail: NidhalAbdulaziz@uowdubai.ac.ae
}

\author{
Eesa Bastaki \\ Department of Technology and Development \\ Dubai Silicon Oasis \\ P O Box 491, Dubai, UAE \\ Tel:+971 4 2027742; Fax: +971 42994116 \\ e-mail: eesa@dso.ae
}

\begin{abstract}
A novel slope line code for data transmission and storage on digital communication systems has been proposed. The new line code operates on the principle of slope coding. The slope encoder transmits alternative slopes (stair-step-like pulses) for the transmission of the $1 \mathrm{~s}$ and $0 \mathrm{~s}$ of the input binary data. The decoder detects the received signal using correlative slope technique in order to extract the transmitted binary $1 \mathrm{~s}$ and $0 \mathrm{~s}$ from the incoming symbols. The encoder and decoder operation were described. The encoder circuit is designed and simulated using MultiSim software. The results show a thorough match with the theory. The new line code has many desirable properties which makes it attractive and a suitable for data transmission and storage on different types of telecommunication networks and multimedia.
\end{abstract}

Index Terms - Data Transmission, Line Codes, Simulation and Modelling, Telecommunication Networks.

\section{INTRODUCTION}

The recent development in telecommunication networks requires efficiency upgrading for the transmission and storage of the different types of information such as data, voice, images, multimedia and real-time video.

The efficiency can be considered from several point of view such as the access and routing protocols, encoding and modulation techniques, system design complexity, cost and many others.

One of these important efficiency parameters is the coding which is thoroughly investigated in the literature.
Many codes have been proposed and then deployed as standards in telecommunication and computer networks.

In general, the coding techniques can be classified into three different categories. These are the source, error control, and line codes, which share some common features between them. The first and the second are not the main topic of this research proposal. The source code is used to remove redundancy from the message while the error control code is used to provide error control over the transmission channels/media.

Line codes are the third category which are used since the introduction of the old telephone systems. They are used in many applications for data storage in multimedia systems and telecommunication networks. These networks can be wire-connected such as local-, metropolitain-, and wide-area networks (LANs, MANs, and WANs), wireless-connected such as wireless LANs and generic wireless telecommunication systems, and optical transmission systems.

Line codes have many desirable properties which give preference to the usage of one line code on another. The desirable properties involve spectrum occupancy with no dc content, self-clocking information, power requirement, coding efficiency, transparency, error detection and correction capabilities, encoding and decoding complexities with their circuits, and finally, the cost of circuitry.

A survey of the principle of operation, properties and applications of line codes may be sought from [1]-[3]. The most recent guide which involves the newly emerging technology and applications in multimedia 
systems, telecommunication and computer networks, and wireless systems may be sought from [4]-[7].

This paper reports on a new line code, which operates on the principle of slope coding. Slope coding is technique in which the slope of the reshaped transmitted/stored pulse is used to carry the information of the binary bit ( 1 or 0$)$. The delta modulation is considered as the oldest technique followed by the Manchester line code. The new line code however uses a multilevel slope coding technique in order to minimise the code power spectral density and provide some other desirable features that are mentioned earlier.

The paper is divided into many sections. It starts with an introduction section. This is then followed by section two which describes the line code encoding and decoding principles. Section three is devoted for the encoder circuit design and its operational performance evaluation. Section four is dealing with the discussion and comparison and further works to be carried out. Finally, the conclusion is given in section five.

\section{ENCODING AND DECODING TECHNIQUES}

The new line coding technique operates on the principle of slope or stair-step encoding. The encoder transmits either positive or negative pulse (slope). These two slopes are decoded at the receiving end to extract the original binary of $1 \mathrm{~s}$ and $0 \mathrm{~s}$ and also to drive the clock signal.

\section{A. The Principles of Encoder Operation}

The encoder function is to generate an output pulse for every binary bit it receives with the same bit/symbol duration. The generated pulse is either positive (i.e. has a positive amplitude with respect to the previous pulse) or negative (i.e. has a negative amplitude relative to the previous pulse).

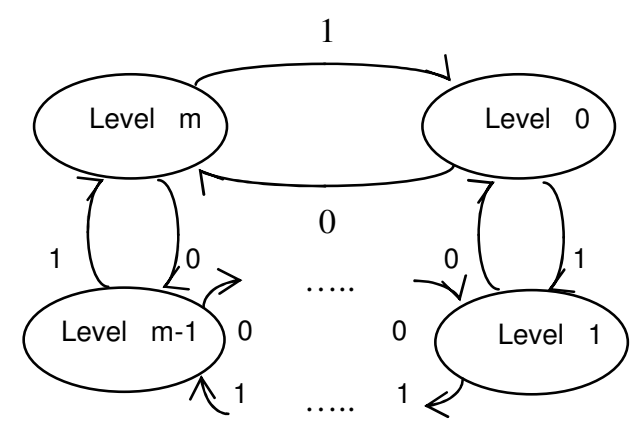

Fig. 1. State transition diagram of the encoder.

Fig. 1 shows the state diagram of the encoder. In the figure, the encoder output is assumed to have $m$ signal levels (symbols). The value of $m$ is an applicationspecific parameter. It can be set to the desired number of encoder output levels. The output levels have direct influence on the shape of the power spectral density of the line code and on its peak-to-average power ratio.

The stair-step/slope line encoder operates as follows.

(1) When a binary 1 is received, the encoder generates a positive going pulse with respect to the previous output symbol with a time duration equivalent to the original binary input data.

(2) When a binary 0 is received, the encoder generates a negative going pulse with respect to the previous output symbol with a time duration that is equivalent to the original binary input data.

(3) In both previous cases, when the encoder output level reaches the two extreme limits of the output $m$ levels, the encoder refreshes its output with a symbol having either the maximum or the minimum level. This means for a run of binary $1 \mathrm{~s}$ at the encoder input, when the encoder output reaches the maximum (level $\mathrm{m}$ ) level. In this case, the decoder switches back to the minimum (level 0) level and then generates a positivegoing symbol in a stair-step form. The same procedure will happen for a run of 0 s of a binary data.

Fig. 2 shows the stair-step- or slope-encoded waveform for an arbitrary input binary sequence of 0 s and $1 \mathrm{~s}$. In this example, it has been assumed that the encoder output has four levels $(m=4)$, two positive (+ve) levels and two negative (-ve) levels. Other values of $m$ can be taken and then the encoded waveforms can be generated accordingly. With $(m=2)$, the encoder output produces a slope-encoded waveform similar to bi-phase (Manchester) code waveform, when a half bit slope decision criterion is introduced. In Fig. 2, other examples of line codes are also given for illustration. Two levels (+ve and -ve) are used to represent the non-return-tozero-level (NRZ-L) and Manchester encoded waveforms. Three levels (+ve, 0, and -ve) are however used for the alternative mark inversion (AMI) or the polar non-returnto-zero waveform representation.

A quick inspection of the slope encoded waveform shows its ability to maintain a transition of signal level at every output symbol. Thus, the waveform has excellent timing information and therefore, it facilitates clock recovery and maintains a strong synchronism between the encoder and decoder. This is perhaps one of the most desirable properties of line codes [1]-[5].

\section{B. The Principles of Decoder Operation}

The decoding operation of the new slope line code is simple as shown in the state diagram in Fig. 3. The incoming multilevel-coded signal undergoes parallel comparison processes with its one-bit delayed version. From the diagram, there are two binary states ( 0 and 1$)$ at the decoder output. The operation of the decoder is as follows. 


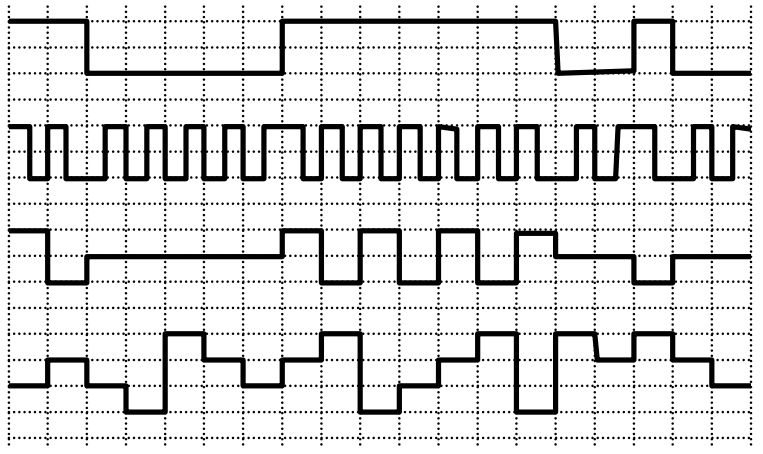

Fig. 2. Example of different encoded waveforms. From top to bottom: NRZ-L, Manchester, AMI, and slope line codes.

(1) When the current output state is binary 0 , then the decoding process for the input m-level signal is carried out depending on the following.

(i) If the signal level is less (negative slope received) in comparison with its delayed version, then it stays in the same state and decode this symbol as an output binary 0 .

(ii) If the signal level is higher (positive slope received), in comparison with its delayed version, then change state to binary 1 , and decode this level as an output binary 1 .

(iii) If there is a positive jump in signal level, where this jump is equal to two levels or more in comparison with its delayed version, then it stays at the same state and decode this symbol as an output binary 0 .

(2) When the current output state is binary 1 , then the decoding process is carried out depending on the following.

(i) If the signal level is higher (positive slope received) in comparison with its delayed version, then it stays at the same state and decodes this symbol as an output binary 1 .

(ii) If the signal level is less (negative slope received) in comparison with its delayed version, then change state to binary 0 , and decodes this symbol as an output binary 0 .

(iii) If there is a negative jump in signal level, where this jump is equal to two levels or more in comparison with its delayed version, then it stays at the same state and decode this symbol as an output binary 1 .

The decoder has a threshold for the comparison process between the received signal and its one-bit delayed version. The threshold level is to be decided depending on the number of levels $(\mathrm{m})$ used at the encoder. This is especially essential when a violation to the rule occurs when there is either a positive or a negative jump in signal level in comparison with the delayed version.

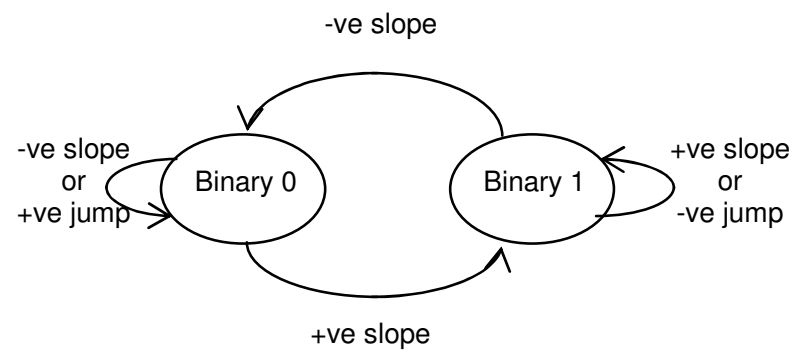

Fig. 3. State transition diagram of the decoder.

\section{ENCODER CIRCUIT DESIGN}

The encoder circuit is designed to have four output levels (i.e. $m=4$ ). This number has been chosen in order to match the example given previously in Fig. 2. A block diagram of the designed circuit is shown in Fig. $4[2,8]$. The 4-level encoder consists of a 2-bit up/down counter and a digital-to-analogue converter (DAC). The encoder is fed with a pseudo-noise (PN) binary data sequence. The PN sequence generator has four stages. Thus, it generates $2^{n}-1=2^{4}-1=15$ pseudo-random binary bits. The counter that it follows is a two-stage up/down counter. Its count is controlled by the input binary sequence. If the input is binary 1 the counter counts up and when the input is binary 0 , it counts down. The output count (2-bit) of the counter is fed to a binary-weighted-input inverting summer amplifier circuit. The latter circuit is a type of a digital-to-analogue converter. The DAC has two inputs that are fed from the outputs of the two-stage up/down counter. The output of the DAC is the required m-level stair-step-/slope-encoded waveform.

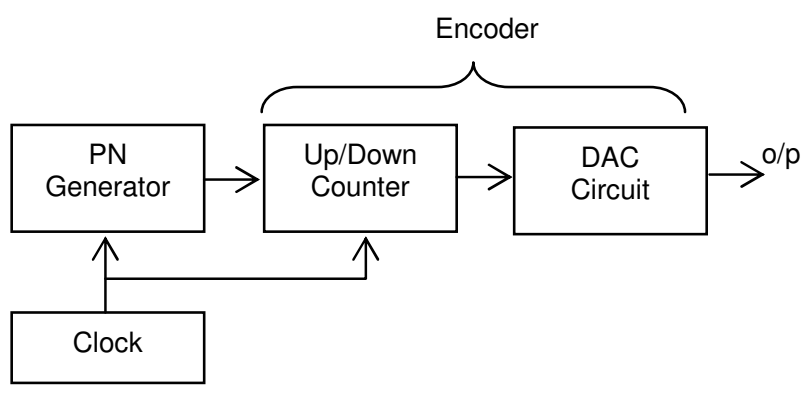

Fig. 4. PN generator and slope encoder block diagram. 


\section{RESULTS}

Fig. 5 shows the results of the output waveforms of the PN binary sequence generator together with the stair-step-/slope-encoded waveforms. The results are obtained from a simulation model that is preformed using Multisim package [9]. The encoded waveform has about a half-bit time delay with respect to the PN sequence data. This is due to the inherent logic circuits propagation delay. The slope-encoded output is also shown inverted in phase due to the use of inverting operational amplifier of the DAC circuit. The encoded waveform starts at an arbitrary level of the four levels that are considered in the design of the encoder circuit.

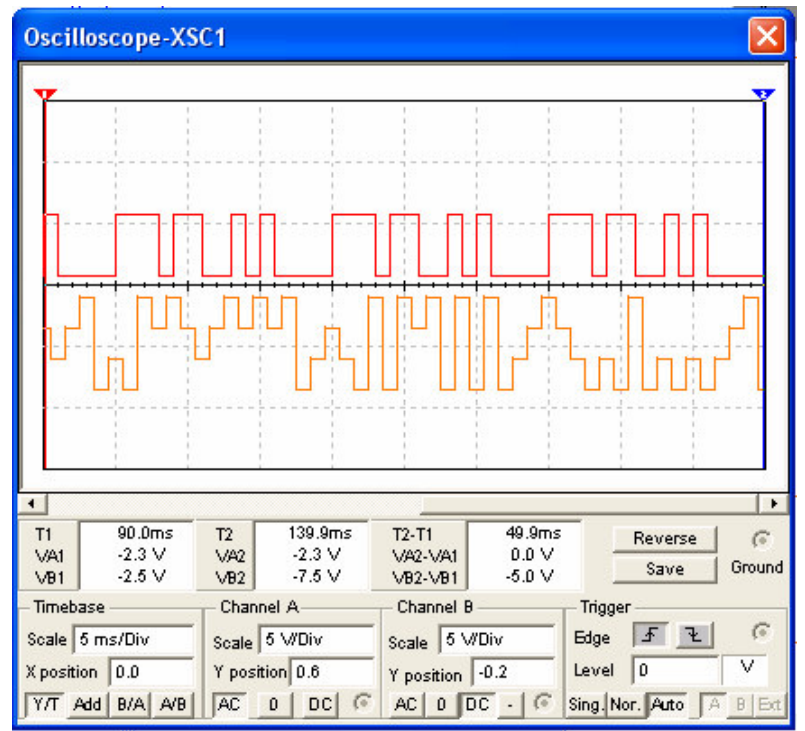

Fig. 5. Slope encoder input (PN sequence) and output (stairstep-/slope-encoded) waveforms.

A higher number of output encoded levels can be obtained with the use of a higher order up/down counter. The DAC circuit which complements the counter satisfactory operation needs in this case an increased number of inputs which matches the parallel bits produced at the output of the up/down counter. The DAC circuit always produces $m=2^{k}$ output levels, where $k$ is the number of stages (bits) the up/down counter has.

\section{DISCUSSION}

Line codes are used extensively in data transmission over telecommunication and computer networks and for data storage in multimedia systems.

The new slope line code proposed here introduces a new generic concept that can be used for encoding messages to any required number of levels. Thus, it has the ability of the inclusion as special cases of some of the binary, ternary and quaternary line codes that have been previously proposed and applied in data transmission. The new line code can generate binary, ternary or quaternary output signal with a special selection of the value of the number of levels. The selection of the number of levels is however governed by two important parameters which are considered very essential in decision about the desirable features of line codes. These are the line code power spectral density and its peak-to-average power ratio. Increasing the number of the slope encoded signal tends to smooth the variation in signal waveform and hence tends to reduce the overall power spectral density of the code. This is considered as one of the most desirable features of line codes. This, however, has the disadvantage of increasing the peak-toaverage power ratio of the line code. An optimisation trade off is thus needed to be set for these two parameters.

The method by which the input binary sequence is slope line encoded is simple and maintains continuous level change of the encoded signal. The continuous change in the encoded signal provides self-clocking information which simplifies clock recovery at the receiving side and thus keeps the encoder-decoder combination in continuous synchronism. This property is also one of the most recommended feature of line codes.

The encoder can be designed for any number of encoded signal levels. In such a case we need to truncate the counter count to match the desired number of output levels. For example for $\mathrm{m}=6$, we need a 3-bit up/down counter that has modulus-6. The DAC circuit in this case should have three inputs. With a 3-bit counter, there are 8 states of which two can be considered as don't care states which simplify the encoder design.

The encoder is still under development for its noise performance evaluation and the quantitative evaluation of its bandwidth occupancy requirement. Finally, further extension to its operation is also under consideration to include the substitutional line codes in its generic operation. This would involve the substitution of x-bit of 1 s or 0 s to represent single output symbol.

\section{CONCLUSIONS}

A new generalised line code has been proposed in this paper. The line code has an $m$-level output. The value of $m$ and hence the number of output levels of the encoded message can be chosen according to a specific application. The operation procedure of the encoder and decoder are well explained. The encoder circuit is designed for only one case that has four output levels. A $\mathrm{PN}$ sequence generator is used to mimic the data that needs to be encoded. The result of the encoder output together with the PN sequence waveforms are given. The results obtained from simulation are in a complete match with the theory. Additional features of the new line code are discussed and compared with other existing line codes. Moreover, a suggestion for further development of the proposal is also given. 


\section{REFERENCES}

[1] W. Cattermole, " Principles of digital line coding," International J. of Electronics, vol. 55, no. 1, pp. 333, 1983.

[2] L. W. Couch, Digital and analog communication systems $\left(5^{\text {th }}\right.$ Ed), Prentice Hall, pp. 127-225, 1997.

[3] J. LoCicero and B. Patel, Line coding, in The Communication Handbook, (J. Gibson), CRC Press, Florida 1996, pp. 386-403.

[4] F. Xiong, Digital modulation techniques, Artech House, pp. 17-83, 2000.

[5] H. Dutton and P. Lenhard, High-speed networking technology: an introductory survey, Prentice Hall, pp. 2/1-51, 1995.
[6] W. Stalling, Data and computer communications $\left(5^{\text {th }}\right.$ Ed), Prentice Hall, New Jersey, 1997, pps. 95-137 and 401-464.

[7] A. Glass and E. Bastaki, "H-Ternary line code for data transmission," International Conference on Communications, Computer and Power (ICCCP'01), Sultan Qaboos University, Muscat, Oman, 12-14 February 2001, pp. 107-110.

[8] T. Floyd, "Digital fundamentals ( $\left.6^{\text {th }} \mathrm{Ed}\right)$ ", Prentice Hall, New Jersey, 1997.

[9] Electronics Workbench Corporation, MultiSIM 2001. Available on line at: http://www.electronicsworkbenck.com (accessed June 2005). 